\title{
THYROID DYSFUNCTION IN WOMEN WITH MENOPAUSAL SYMPTOMS
}

\author{
Omnia State*, Hesham Shalan, Soma Sherief** \\ Department of Obstetrics \& Gynecology, Department of General Medicine* and \\ Department of Clinical Pathology**, Mansoura University, Egypt
}

\section{ABSTRACT}

Background : Many of the menopausal manifestations resemble those attributed to thyroid hypofunction or even hyperfunction. This study aimed at recognizing the role that might be played by the thyroid disturbances in such symptoms.

Materials \& Methods: The study comprised 135 women with menopausal symptoms attending the gynecologic outpatient clinic and the endocrinology outpatient clinic at Mansoura University hospitals. All women had serum TSH, $\mathrm{T}_{3}$ and $\mathrm{T}_{4}$ tested.

Results : Among the menopausal women in this study, 11 women (8.1\%) sufferred thyroid dysfunction; 6 women (4.4\%) with thyroid hypofunction suffered cognitive and moodal changes and neurological manifestations as paraesthesia and muscle cramps. The other 5 women $(3.7 \%)$ with thyroid hyperfunction, mostly suffered hot flushes and depression. Nevertheless, all the differences were statistically insignificant.

Conclusion : From these preliminary results, it is believed that postmenopausal women with severe or resistant menopausal symptoms should be offered $\mathrm{TSH}, \mathrm{T}_{3}$ and $\mathrm{T}_{4}$ assays to rule out the thyroid disturbances before attempting hormone replacement therapy.

Key words : Postmenopausal women, menopausal symptoms, thyroid dysfunction.

\section{INTRODUCTION}

Throughout recorded history, multiple physical and mental conditions have been attributed to the menopause. The belief that somatic and behavioral disturbances are related to the female reproductive changes is an ancient one that persists to the contemporary times. However, there are changes in other endocrinal functions that are undoubtedly sharing in these disturbances ${ }^{(1-3)}$.

The thyroid is one of the major endocrinal glands that affect all body functions. However, thyroid gland dysfunction is a relatively common clinical problem in elderly people and is associated with significatnt morbidity if left untreated. The clinical features of thyroid disease may be subtle, easily overlooked or misdiagnosed (4-6). Therefore, a high index of suspicion is necessary. If potentially serious sequelae are to be avoided, the selection and interpretation of thyroid function tests must be appropriate. It is particularly important to consider both the effect of concurrent illness and the effect of certian drugs on thyroid

Corresponding author: Hesham Shalan, Department of OB/GYN, Faculty of Medicine, Mansoura University

E-mail drshalan@forislam.com 
function tests. With recent methodological advances, thyroid function tests are now more reliable, though in certain situations they still need to be interpreted with caution. Once the diagnosis is established, the management of both hypothyroidism and hyperthyroidism is relativley simple and effective, though there are special considerations relating to elderly patients $(6,7)$.

Several studies investigating the thyroid function in menopausal women failed to demonstrate remarkable age-related changes ${ }^{(9-11)}$. It has been established that $\mathrm{T}_{4}$ levels remain normal through homeostatic mechanisms. Production of $\mathrm{T}_{4}$ decreases by $25 \%$ with age and metabolic clearance of $\mathrm{T}_{4}$ is reduced. Conversion of $\mathrm{T}_{4}$ to $\mathrm{T}_{3}$ decreases and $\mathrm{TSH}$ levels increase. Plasms thyroid-binding globulins are also unchanged in healthy individuals (12-15).

Many of the menopausal manifestations namely hot flushes, moodal changes, gastrointestinal changes and cardio-vascular changes resemble those attributed to thyroid hyperfunction or even hypofunction $(16,17)$. Because physical signs and symptoms of thyroid dysfunction are less reliable in the elderly, serum testing for confirmation of disease is necessary in the evaluation of suspected hypothyroidism and hyperthyroidism $(18,19)$. this study aimed at investigating menopausal women suffering menopausal symptoms in an attempt to recognize the role that might be played by the thyroid disturbances in such symptoms.

\section{MATERIALS \& METHODS}

The study comprised 135 women with documented menopause and presented to the gynecologic outpatient clinic and the outpatient clinic of the Endocrinology Unit at Mansoura University Hospitals, with different menopausal symptoms (Table I). All women in the study had history of secondary amenorrhoea for at least 1 year and serum FSH above $20 \mathrm{m \mu} / \mathrm{mL}$ before being included in the study to document the menopausal state.

All women were subjected to thorough history taking and physical examination and all other necessary investigations that the situation might call for. All women with any apparent medical problems that might be contributing to the presenting symptoms were excluded from the study. All women were then subjected to testing for serum $\mathrm{T}_{3}, \mathrm{~T}_{4}, \mathrm{TSH}$. Women who were found to have thyroid dysfunction were treated properly and the other women were subjected to treatment of their menopausal symptoms with estrogen replacement therapy when appropriate. Student $T$ test was used for analysis of difference.

\section{RESULTS}

Table (I) is demonstrating the postmenopausal women characteristics. The Women had different durations since the onset of the menopause. Hot flushes were the commonest symptoms in the study group. figure (1) is representing the thyroid function in the study group and shows that 11 women $(8.1 \%)$ sufferred thyroid dysfunction; 6 women $(4.4 \%)$ sufferred thyroid hypofunction and 5 women $(3.7 \%)$ thyroid hyperfunction. Table (II) shows the thyroid function in relation to menopausal symptoms. Most of the women with thyroid hypofunction sufferred cognitive and moodal changes and neurological manifestations as paraesthesia and muscle cramps. Women with thyroid hyperfunction mostly suffered hot flushes and depression. Nevertheless, all differences were statistically insignificant. 
Table I : The characteristics of the postmenopausal women $(n=135)$

\begin{tabular}{|l|c|}
\hline \multicolumn{1}{|c|}{ Variable } & Mean \pm S. D. \\
\hline - Age (years) & $60.5( \pm 6.67)$ \\
- Gravidity & $6.3( \pm 2.1)$ \\
- Parity & $4.5( \pm 2.2)$ \\
- Body mass index & $26.6( \pm 3.4)$ \\
- Menopausal years & $11.5( \pm 3.17)$ \\
- Menopausal symptoms * & \\
- Hot flushes & $90(66.66 \%)$ \\
- Cognitive \& moodal changes & $44(32.59 \%)$ \\
- Urinary symptoms & $33(24.44 \%)$ \\
- Sexual dysfunction & $23(17.03 \%)$ \\
\hline
\end{tabular}

* Some women prescnted by more than one symptom.

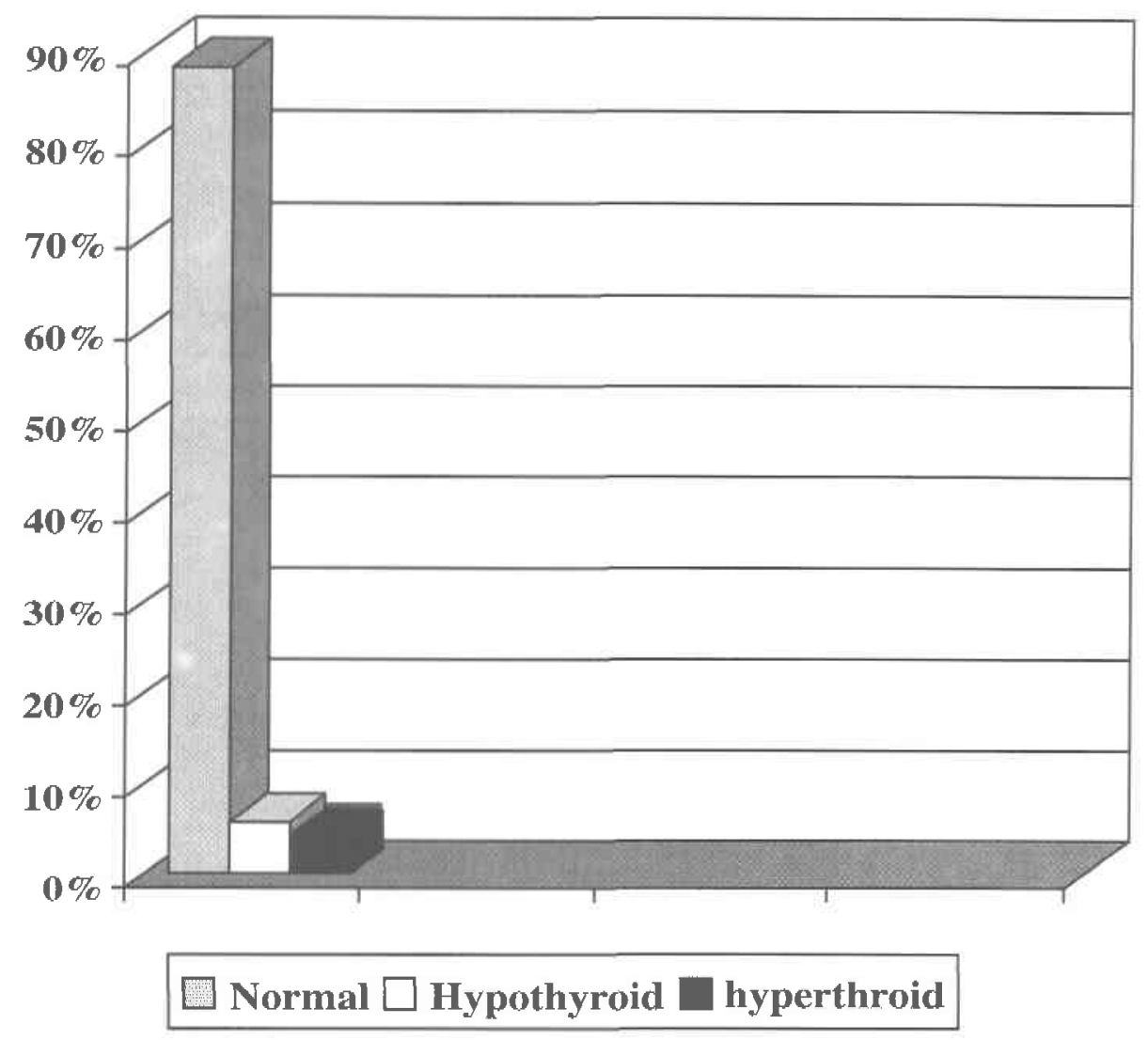

Fig. 1. Thyroid lunction of the studied postmenopausal women 
Table II : Thyroid function in relation to the presenting symptoms

\begin{tabular}{|l|c|c|c|c|}
\hline \multicolumn{1}{|c|}{ Complaint } & N & Normal & Hypothyroid & Hyperfunction \\
\hline Hot flushes* & 90 & $85(62.96 \%)$ & 0 & $5(3.70 \%)$ \\
Cognitive \& moodal changes * & 44 & $36(26.67 \%)$ & $5(3.70 \%)$ & $3(2.22 \%)$ \\
Urinary symptoms * & 33 & $31(22.69 \%)$ & $2(1.48 \%)$ & 0 \\
Sexual dysfunction * & 23 & $19(14.07 \%)$ & $3(2.22 \%)$ & $1(0.74 \%)$ \\
\hline
\end{tabular}

* All differences were statistically insignificant $(\mathrm{P}<0.05)$

\section{DISCUSSION}

Thyroid dysfunction is not uncommon in elderly women. Undoubtedly, there is similarity in presentation between the menopausal symptoms and hypothyroidism and hyperthyroidism as the hot flushes, cognitive and moodal changes, gastrointestinal and genitourinary symptoms $(2,18,19)$. Should the thyroid function be tested in menopausal women suffering from severe menopausal symptoms? was the question that the study was planned to answer.

In this study, there were 6 women $(4.4 \%)$ with serum $\mathrm{TSH}>10 \mu \mathrm{U} / \mathrm{mL}$. These results were similar to others, who reported a prevalence of definite hypothyroidism of $5.9 \%$ in postmenopausal women (20). Another study reported $5.9 \%$ of their women had borderline elevation of TSH (5 to $10 \mu \mathrm{U} / \mathrm{mL}$ ), with $12.7 \%$ of these having low serum $T_{4}{ }^{(13)}$. Other prevalence studies showed different results among their populations ranging from $0.5 \%$ to $17.5 \%$. However, these studies were using different selection criteria rendering direa comparisons difficult $(21,22)$,
The most common causes of hypothyroidism in the elderly are autoimmune thyroiditis or previous treatment for Graves' disease. Only $10 \%$ to $20 \%$ of patients with laboratory-determined hypothyroidism are recognized on clinical examination $(4,6)$. Complaints are subtle and difficult to distinguish from symptoms associated with aging and menopause ${ }^{(1,18)}$. Fatigue $(67.7 \%)$ and weakness $(52.5 \%)$ were the two most common complaints in elderly women above 70 years with documented hypothyroidism. Mental slowness $(45.3 \%)$, drowsiness $(39.7 \%)$, chilliness $(34.9 \%)$, dry skin $(34.5 \%)$, constipation $(32.8 \%)$, and deafness $(32.1 \%)$ were found in less than hall of subjects. In another study conducted on a group of young $(<55$ years-old $)$ hypothyroid patients, clilliness, paresthesias, weight gain, or muscle cramps were seen significantly less often (23).

In this study, 5 women $(3.7 \%$ ) had low TSH and high $\mathrm{T}_{4}$ levels. The prevalence of hyperthyroidism in the elderly women ranges from $0.5 \%$ to $2.3 \%$ (2.5) depending on the criteria for diagnosis and the population studied. Symptoms of hyperthyroidism, like those of hypothyroidism, can be subtle and difficult to detect in the elderly $(6,8)$. Possible manifestations include depression, change 
in bowel habits, chronic fatigue, emotional liability, and muscle weakness and wasting. The classic symptoms of thyrotoxicosis are seen in only one third of patients aged more than 70 years (24). the most common signs are tachycardia, fine skin, tremor, atrial fibrillation, and hyperactive reflexes. Toxic multinodular goiter is the most common cause of hyperthyroidism in the elederly; the incidence of Graves' disease declines after 60 years of age ${ }^{(9)}$. Iodine-induced hyperthyroidism (jodbasedow phenomenon) is seen in patients with nontoxic multinodualr goiter after the excessive intake of iodine or iodine-containing substances $(25,26)$

High-sensitivity TSH assays have simplified the diagnosis of thyrotoxicosis. A suppressed TSH level in association with elevated $\mathrm{T}_{3}$ or $\mathrm{T}_{4}$ is generally diagnostic of hyperthyroidism. However, an isolated low TSH level in postmenopausal women does not have a high predictive value for hyperthyroidism. Decreased serum cholesterol, elevated alkaline phosphatase, and mildly increased serum calcium are occasionally found in thyrotoxicosis $(26,27)$.

Despite the prevalence of thyroid dysfunction in postmenopausal women, there is often considerable dealy and difficulty in diagnosis because symptoms are subtle and attributed to normal aging. Furthermore, atypical presentations are common. Consequently, physicians should maintain a high index of suspicion and have a low threshold for testing thyroid function. Subclinical abnormalities of thyroid function are even more prevalent than overt disease ${ }^{(6,8)}$. The treatment should be individualized as the elderly are vulnerable to the side effects of thyroid and anti-thyroid medications, which mandates cautious use of drugs and careful dose adjustments ${ }^{(6,8)}$.
In this study, women with proved thyroid deysfunction were treated properly and this led to relief of their complaint. Estrogen replacement therapy (ERT) is not without complications and although this is a very controversial issue, women will prefer to have their symptoms relieved without resorting to ERT. Again, ERT and other non-specific treatments become ineffective untill the thyroid disturbances are properly corrected. We believe that postmenopausal women with severe or resistant menopausal symnptoms should be offered TSH, $\mathrm{T}_{3}$ and $\mathrm{T}_{4}$ assays to rule out the thyroid disturbances before attempting hormone replacement therapy.

\section{REFERENCES}

1- Eaton SE, Webster J, Allahabadia A. Thyroid disease and the menopausal woman. J Br Menopause Soc 2003; 9(2): 82-4.

2- Schindler AE. Thyroid function and postmenopause. Gynecol Endocrinol. 2003; 17(1): 79-85.

3- Samuels $\mathrm{MH}$. Subclinical thyroid disease in the elderly. thyroid 1998; 8(9): 803-13.

4- Vanderpump MP, Tunbridge WM. Epidemiology and prevention of clinical and subclinical hypothyroidism. Thyroid 2002; 12(10): 839-47.

5- Chuo AM, Lim JK. Thyroid dysfunction in elderly patients. Ann Acad Med Singapore, 2003; 32(1): 96-100.

6- Wallace K, Hofmann MT. Thyroid dysfunction: how to manage overt and subclinical disease in older patients. Geriatrics. 1998; 53(4): 32-8.

7. Mohandas R, Gupta KL. Managing thyroid dysfunction in the elderly. Answers to seven common questions. Postgrad Med. 2003; 113(5); 54-6.

8- Finucane P, Anderson C. Thyroid disease in older 
patients. Diagnosis and treatment. Drugs Aging. 1995; 6(4): 268-77.

9- Li TM. Hypothyroidism in elderly people. Geriatr Nurs. $2002 ; 23(2)$ : 88-93.

10- Fatourechi V. Subclinical thyroid disease. Mayo Clin Proc. 2001; 76(4): 413-6.

11- Faughnan M, Lepage R, Fugere P, Bissonnette F, Brossard JH, D'Amour P. Screening for thyroid disease at the menopausal clinic. Clin Invest Med. $1995 ; 18(1): 11-8$.

12- Sowers M, Luborsky J, Perdue C, Araujo KL, Goldman MB, Harlow SD. Thyroid stimulating hormone (TSH) concentrations and menopausal status in women at the mid-life. Clin Endocrinol $2003 ; 58(3): 340-7$

13- Sawin CT, Castelli WP, Hershman JM. The aging thyroid: thyroid deficiency in the Framingham study. Arch Intern Med 1985; 145; 1386.

14- Sawin CT, Geller A, Kaplan MM. Low serum thyrotropin (thyroid stimulating hormone) in older persons without hyperthyroidism. Arch Intern Med 1991; 151: 165 .

15- Sawin CT, Herman T, Molitch ME. Aging and the thyroid: Decreased requirement for thyroid hormone in older hypothyroid patients. Am J Med 1983; 75: 206.

16- Nelson HD. Commonly used types of estrogen for treatment of hot flushes: Scientific review. JAMA $2004 ; 7,291(13): 1610-20$.

17- Grodstein F, Lifford K, Ronick NM, Curhan GC. Postmenopausal hormone replacement therapy and risk of urinary incontinence. obstet Gynecol 2004; I03(2): 254-60.

18- Surks MI, Ocampo E. Subclinical thyroid disease. Am J Med. 1996; 100(2): 217-23.

19- Costante G, Crocetti U, Schifino E, Ludovico O, Capula $\mathrm{C}$, Nicotera $\mathrm{M}$, et al. Slow growth of benign thyroid nodules after menopause: no need for long-term thyroxine suppressive therapy in post-menopausal women. J Endorcrinol Invest. $2004 ; 27(1): 31-6$.

20- Wilson MM. Menopause. Clin geriar Med. 2003; 19(3): 483-506

21- Levy EG. Thyroid disease in the elderly. Med Clin North Am 1992; 75(1): 151-67.

22- Suchartwatnachai $\mathrm{C}$, Theppisai $\mathrm{U}$, Jirapinyo $\mathrm{M}$ Screening for hypothyroidism at a menopause clinic. Int J Gyn Obstet 2002; 77(1): 39-40.

23- Doucet J, Trivalle C, Chassagne P. Does age play a role in clinical presentation of hypothyroidism? J Am Geriatr Soc 1994; 42(9): 984-6.

24- Trivalle $\mathrm{C}$, Doucet $\mathrm{J}$, Chassagne $\mathrm{P}$. differences in the signs and symptoms of hyperthyroidism in older and younger patients. J Am geriatr Soc 1996; 44(1): 50-3.

25- Leger A, Massin J, Laurent M. Iodine-induced thyrotoxicosis : An analysis of eighty-five consecutive cases. Fur J Clin Invest 1984; 14(6): 449-55.

26- Levin R. Thyrotoxicosis in the elderly. J Am Geriatr Soc $1987 ; 35: 587-9$.

27- Duntas LH. Thyroid disease and lipids. Thyroid $2002 ; 12(4): 287-93$. 